\title{
Adaptive Generalized Gaussian Distribution Oriented Thresholding Function for Image De-Noising
}

\author{
Noorbakhsh Amiri Golilarz ${ }^{1}$, Hasan Demirel $^{2}$, Hui Gao ${ }^{3}$ \\ School of Computer Science and Engineering \\ University of Electronic Science and Technology of China, Chengdu, China ${ }^{1,3}$ \\ Department of Electrical and Electronic Engineering, Eastern Mediterranean University, Cyprus ${ }^{2}$
}

\begin{abstract}
In this paper, an Adaptive Generalized Gaussian Distribution (AGGD) oriented thresholding function for image de-noising is proposed. This technique utilizes a unique threshold function derived from the generalized Gaussian function obtained from the HH sub-band in the wavelet domain. Twodimensional discrete wavelet transform is used to generate the decomposition. Having the threshold function formed by using the distribution of the high frequency wavelet $\mathrm{HH}$ coefficients makes the function data dependent, hence adaptive to the input image to be de-noised. Thresholding is performed in the high frequency sub-bands of the wavelet transform in the interval [-t, $t$ ], where $t$ is calculated in terms of the standard deviation of the coefficients in the $\mathrm{HH}$ sub-band. After thresholding, inverse wavelet transform is applied to generate the final de-noised image. Experimental results show the superiority of the proposed technique over other alternative state-of-the-art methods in the literature.
\end{abstract}

Keywords-Adaptive generalized Gaussian distribution; thresholding function; image de-noising; high frequency sub-bands

\section{INTRODUCTION}

Noise can corrupt the image through acquisition or transmission processes. The main objective in image denoising is to eliminate or reduce the level of noise to enhance the visual quality of image.

Wavelet based image de-noising has become very popular among other noise removing techniques. Applying wavelet transform leads to two types of coefficients which can be divided into important and non-important coefficients, with the former should be kept due to having the most important characteristics of the image and the latter should be discarded.

Therefore, noise suppression in wavelet domain requires a suitable threshold value to remove small noisy components of high frequency sub-bands and preserve larger coefficients of the same sub-bands. In this regards, an appropriate thresholding function and a defined threshold value are needed to suppress the additive noise and keep the noise-free data.

In this study, AGGD oriented thresholding function for image de-noising is proposed. The proposed method is unique such that it generates data dependent thresholding function for each noisy image. This method is very significant in removing small noisy coefficients in the interval $[-t, t]$. Here the denoising results of the proposed method are compared with some alternative techniques to show the superiority of the proposed method. Experimental results show that the proposed method obtains up to $2.66 \mathrm{~dB}$ PSNR improvement over the state-of-the-art for de-noising Barbara image.

\section{RELATED WORKS}

Many methods have been done to discard the noise from images using wavelet transform. G. Y. Chen et al., in [1] proposed neighbor dependency and customized wavelet and threshold. N. A. Golilarz and H. Demirel utilized TNN with smooth sigmoid based shrinkage function (SSBS) for image de-noising [2]. Adapting to unknown smoothness via wavelet shrinkage and ideal spatial adaptation by wavelet shrinkage is proposed by Donoho and Johnstone in [3] and [4], respectively. J. Portilla et al., in [5] proposed de-noising by scale mixture of Gaussians in the wavelet domain. Sveinsson and Benediktsson in [6] proposed almost translation invariant wavelet transformations for speckle reduction of images.

De-noising using smooth nonlinear soft thresholding function is introduced in [7]. Chang, Yu and Vetterli in [8] used adaptive wavelet thresholding for image de-noising. In [9] de-noising by soft thresholding is proposed by Donoho. Also, Coifman in [10] proposed translation invariant method for wavelet based image de-noising. In 2002, Sendur and Selesnick have introduced a wavelet based bivariate shrinkage for image de-noising [11]. De-noising using un-decimated wavelet transform [12] and TNN based noise reduction with a new improved thresholding function [13] are also proposed to discard the noise and improve the quality of images.

\section{WAVELET TRANSFORM}

Function $\mathrm{X}(\mathrm{t})$ can be expanded in terms of scale function $\emptyset(t)$ and wavelet function $\psi(t)$ [14]. One dimensional discrete wavelet transform (DWT) can be written as the following [14] are scale and wavelet functions, respectively and the inner products $a c_{j, k}=\left\langle X, \emptyset_{j, k}\right\rangle, d c_{j, k}=\left\langle X, \psi_{j, k}\right\rangle$ are scaling and wavelet coefficients, respectively.

$X(t)=\sum_{k} a c_{j 0, k} \emptyset_{j 0, k}+\sum_{j \leq j_{0}} \sum_{k} d c_{j, k} \psi_{j, k}$

Where

$\emptyset_{j, k}=2^{j} \emptyset\left(2^{j} t-k\right), \quad \psi_{j, k}=2^{j / 2} \psi\left(2^{j} t-k\right)$

In discrete wavelet transform (DWT), input signal passes through low pass and high pass filters followed by decimation. Then, DWT decomposes the input signal in detail and approximation coefficients. Passing signal through low pass filter, discards all high frequencies. Filtering is followed by 
down sampling. By filtering (low pass filter) and then subsampling, half of the frequencies will be discarded. Hence the resolution is halved after low pass filter (level one). The process continues in level two, where the output of the low pass filter is subsampled by 2 after high pass and low pass filtering again with half of the previous cut off frequencies. In further levels the same process is repeated. In addition, in the higher dimensional discrete wavelet transforms like 2D-DWT, the decomposition for one level is generated by applying 1DDWT on both rows and columns [14]. Thus, we get four sub bands, where three sub bands correspond to high frequencies $\mathrm{HH}, \mathrm{LH}$ and $\mathrm{HL}$ and one sub band includes low frequency, LL. In this paper, higher levels of decomposition are generated by decomposing LL sub band in two dimensional wavelet transform (2D-DWT).

\section{The Procedure of IMAGE DE-NOISING BASED ON WAVELET TRANSFORM}

Applying DWT on an image provides us with wavelet coefficients falling into different sub-bands. Wavelet components can be categorized in two ways: one is wavelet coefficients carrying negligible noise component and other coefficients carrying dominant noise components. It is obvious that, it is required to suppress the noise by selecting a proper threshold value [9]. Proceeding step is setting a threshold value to see which coefficients are within the interval characterized by the threshold value and which coefficients are beyond this interval. Coefficients within the magnitude interval of this threshold value are killed, while the ones beyond this interval are kept/shrunk by thresholding function. The last step is applying inverse discrete wavelet transform to reconstruct the image from thresholded wavelet coefficients.

\section{Proposed Image De-Noising TeChniQue}

In this paper, wavelet based image de-noising using a data driven thresholding function is utilized. Discrete wavelet transform (DWT) is used to decompose the noisy input images into four wavelet sub-bands: $\mathrm{HH}, \mathrm{HL}, \mathrm{LH}$ and LL. Considering the high frequency characteristic of the additive noise, the proposed thresholding function is applied only on high frequency sub-bands $\mathrm{HH}, \mathrm{HL}$ and $\mathrm{LH}$. High frequency sub-bands go through thresholding assuming that noise is suppressed in the thresholded wavelet coefficients. Then inverse wavelet transform is applied on thresholded coefficients to reconstruct the de-noised image. Here 'sym4' wavelet function with four levels of decomposition is used.

It is very important to use an appropriate thresholding function. Many researches introduced different thresholding functions namely hard thresholding, soft thresholding, improved hard and improved soft thresholding functions. The thresholding function is the utmost aspect of a de-noising process. In addition to function, the interval of thresholding is also crucial to perform the most effective de-noising process.

The main focus in applying thresholding is keeping larger coefficients corresponding to the actual signal forming the image and getting rid of very small coefficients generally representing the noise. Hard thresholding, operating in an interval of $[-\mathrm{t}, \mathrm{t}]$ suppresses the noise by preserving larger coefficients and killing small coefficients. On the other hand, soft thresholding operates in the same interval in the same way. However, the larger coefficients outside of the interval are shrunk suppressing the high frequency details including the noise. Zhang in [15] proposed an improved soft thresholding function which depends on the parameter lambda $(\lambda)$ that is empirically determined maximizing Peak Signal to Noise Ratio (PSNR). Moreover, Sahraeian in [16] proposed improved version of hard thresholding function to improve the results of Zhang's method. His technique is also based on choosing the $b$ parameter empirically. The main objective of this paper is to formulate a thresholding function free from heuristic consideration and tailor a threshold function which is dependent on the input data. The most important advantage of the proposed threshold function is its ability to adapt to changing images, hence characterizing a thresholding function to the specific noisy image.

\section{A. Generation of the Proposed Adaptive Generalized Gaussian Distribution (AGGD) Oriented Thresholding Function}

The proposed threshold function is powered by the generalized Gaussian distribution extracted from the given noisy image. The robust median estimator, $\sigma_{n}$, which is calculated by using the $\mathrm{HH}$ wavelet coefficients of the level 4 DWT decomposition using "sym4" wavelet function, is used to generate the Gaussian distribution. Robust median estimator, which can be attributed to the standard deviation of the noise is defined in (3).

$\sigma_{n}=\operatorname{Median}\left(\left|D_{x, y}\right|\right) / 0.6745$

Where, $D_{x, y}$ is wavelet coefficients in $\mathrm{HH}$ sub-band of level 4 decomposition. A five steps procedure is employed to generate the proposed thresholding function.

1) Generate $f(x)$ : Zero mean Gaussian distribution function $f(x)$ in (4) is the first step in generating the threshold function.

$f(x)=\frac{1}{\sqrt{2 \pi \sigma_{n}^{2}}} e^{\frac{-x^{2}}{2 \sigma_{n}^{2}}}$

Where, $x$ is wavelet coefficients in $\mathrm{HH}$ subband of level 4 decomposition. Fig. 1(a) illustrates $f(x)$ of noisy image.

2) Generate $p(x)$ : $\mathrm{p}(\mathrm{x})$ given in (5) is piece-wisely defined function, employing positive and negative inverse of $f(x)$ for positive and negative values of $\mathrm{x}$ respectively as follows. Fig. 1(b) illustrates $\mathrm{p}(\mathrm{x})$.

$$
\begin{aligned}
& p(x)=\left\{\begin{aligned}
(f(x))^{-1}, & x \geq 0 \\
-(f(x))^{-1}, & x<0
\end{aligned}\right. \\
& =\left\{\begin{array}{c}
\left(\frac{1}{\sqrt{2 \pi \sigma_{n}^{2}}} e^{\frac{-x^{2}}{2 \sigma_{n}^{2}}}\right)^{-1}, x \geq 0 \\
-\left(\frac{1}{\sqrt{2 \pi \sigma_{n}^{2}}} e^{\frac{-x^{2}}{2 \sigma_{n}^{2}}}\right)^{-1}, x<0
\end{array}\right.
\end{aligned}
$$


Then, $p(x)$ can be alternatively formulated as follows.

$p(x)=\sqrt{2 \pi \sigma_{n}^{2}} e^{\frac{x^{2}}{2 \sigma_{n}^{2}}}$

3) Normalize $p(x)$ : $\mathrm{q}(\mathrm{x})$ is the normalized $\mathrm{p}(\mathrm{x})$ which is generated as follows. The prospective threshold function is a function to be in line by the identity function. In this context, $\mathrm{p}(\mathrm{x})$ should be normalized (scaled down) with a constant $1 / \mathrm{N}$, so that it is in line by the identity function. Hence the following equality is defined.

$q(x)=p(x) \times 1 / N=x$

$\sqrt{2 \pi \sigma_{n}^{2}} e^{\frac{x^{2}}{2 \sigma_{n}^{2}}} \times 1 / N=x$

Taking the derivative of both sides of (8)

$\left(2 x / 2 \sigma_{n}^{2}\right) \times\left(\sqrt{2 \pi \sigma^{2}} e^{\frac{x^{2}}{2 \sigma^{2}}} \times 1 / N\right)=1$

Using (9) we have:

$\left(2 x / 2{\sigma_{n}}^{2}\right) \times x=1$, so $x=\sigma_{n}$

Using (8), (9) and (10), $N$ can be obtained as:

$N=\left(e^{1 / 2} / \sigma_{n}\right) \times \sqrt{2 \pi \sigma_{n}^{2}}$

Finally, the following equation can be written:

$q(x)=\sqrt{2 \pi \sigma_{n}^{2}} e^{\frac{x^{2}}{2 \sigma_{n}^{2}}} \times 1 / N$

$=\sqrt{2 \pi \sigma_{n}^{2}} e^{\frac{x^{2}}{2 \sigma_{n}^{2}}} \times 1 /\left(\left(e^{1 / 2} / \sigma_{n}\right) \times \sqrt{2 \pi \sigma_{n}^{2}}\right)$

$=\left(\frac{1}{\sigma_{n}} e^{\frac{-x^{2}}{2 \sigma_{n}^{2}}+\frac{1}{2}}\right)^{-1}=\sigma_{n} e^{\frac{x^{2}}{2 \sigma_{n}^{2}}-1 / 2}$

Fig. 1(c) illustrates $q(x)$.

4) Generate $\eta(x)$ : The discontinuity at $\mathrm{x}=0$ should be removed by shifting the curve for $x \geq 0$ down and for curve $x<0$ up, respectively. The following equation is formulated for this operation. Fig. 1(d) illustrates $\eta(\mathrm{x})$, which is now continuous.

$\eta(x)=q(x)-q(0)$

5) Generate $h(x)$ : The final thresholding function (14) is a piecewise defined function as follows. $\eta(\mathrm{x})$ defines the function in the interval $[-t, t]$, where the rest of the function outside this interval is defined by the identity function.

$h(x)=\left\{\begin{array}{c}x, x<-t \\ \eta(x),|x| \leq t \\ x, \quad x>t\end{array}\right.$

Where $t$ is the threshold value which can be obtained by using the intersection of the functions $\eta(x)$ and $x$ as can be seen in (15). Fig. 1(e) shows $h(x)$, which illustrates the final form of the proposed thresholding function.

$\sigma_{n}\left(e^{\frac{t^{2}}{2 \sigma_{n}^{2}}-1 / 2}-e^{-1 / 2}\right)=t$

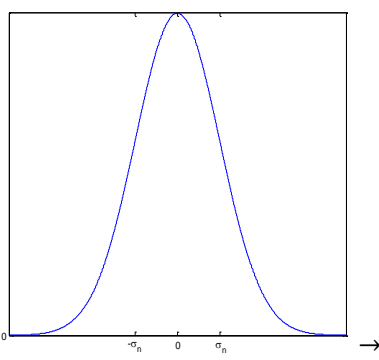

(a)

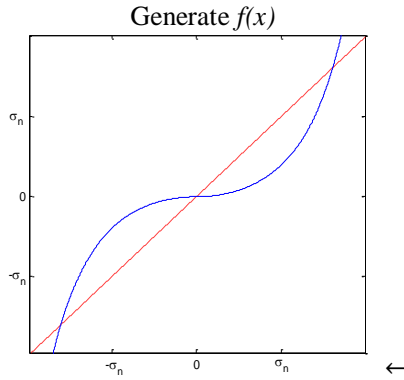

(d)

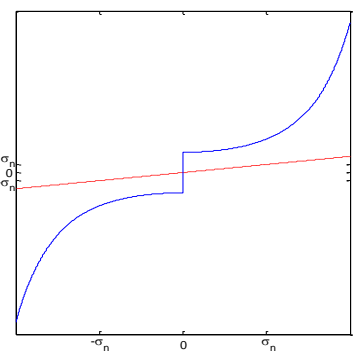

(b)

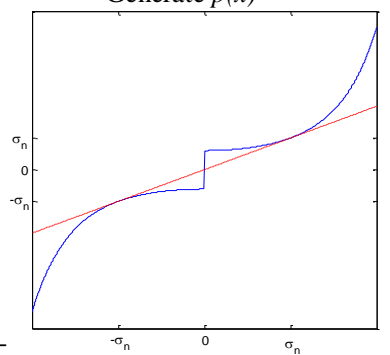

(c) Generate $q(x)$ Generate $p(x)$

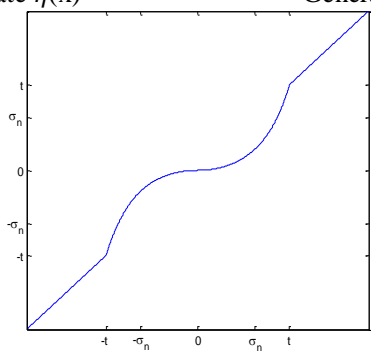

(e)

Generate $h(x)$

Fig. 1. The Process of Obtaining the Proposed Function $h(x)$. The Red Line is the Identity Function.

\section{B. The Analysis of the Proposed Thresholding Function}

Fig. 2 shows hard, soft, improved-hard (by Sahraeian with $b=0.1$ [16]), improved-soft (by Zhang with $\lambda=0.01$ ) and proposed thresholding function. The improved soft threshold function proposed by Zhang [15] is controlled by parameter $\lambda$. This parameter is tuned according to the data to be processed. For example, the parameter $\lambda$ in Fig. 2 is drawn for the value of 0.01 , which is the optimal value leading to highest PSNR in [15]. Same approach is employed in Sahraeian's improved hard thresholding technique which depends on a parameter $b$ that is chosen empirically. In [16] he utilized $b=0.1$ as the optimal value generating highest PSNR. It is clear that $\lambda=0.01$ and $b=0.1$ are best fits in shaping the transformation function leading to thresholding of wavelet coefficients for de-noising in Zhang and Sahraeian's method, respectively. It is obvious that an alternative approach where no empirical parameter optimization process is required and also free from the training samples would be ideal. In this regard, the proposed thresholding function is free from empirical parameter consideration. Furthermore, the proposed thresholding function goes through a data-specific process to model its shape according to the distribution of the input noisy signal to be de-noised. This adaptive process is the most important novelty of the proposed thresholding function. 
One of the most important advantages of the proposed thresholding function is that, it is data dependent, where the data is coming from the diagonal wavelet sub-band (i.e. HH sub-band) after 4 levels of decomposition of the input noisy image. This process generates a dedicated threshold function for every different input noisy image. Fig. 3 illustrates generalized Gaussian distribution for 'Lena', 'Barbara', 'Boat' and 'Mandrill' images. Fig. 4 shows the proposed thresholding functions corresponding to four noisy images with changing frequency characteristics. In this context, we used 'Lena', 'Barbara', 'Boat' and 'Mandrill' images. 'Lena' is an image having more low frequency components while 'Mandrill' has high frequency components. As can be seen in Fig. 4, the proposed thresholding function is changing from image to image. This is due to respective dependency to $\sigma_{n}$ for different noisy images.

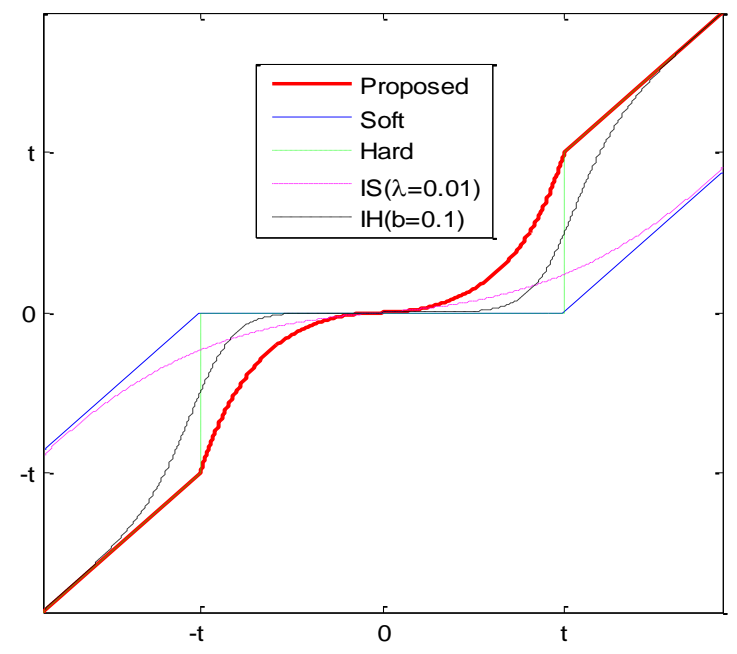

Fig. 2. Proposed Versus Alternative Thresholding Functions.

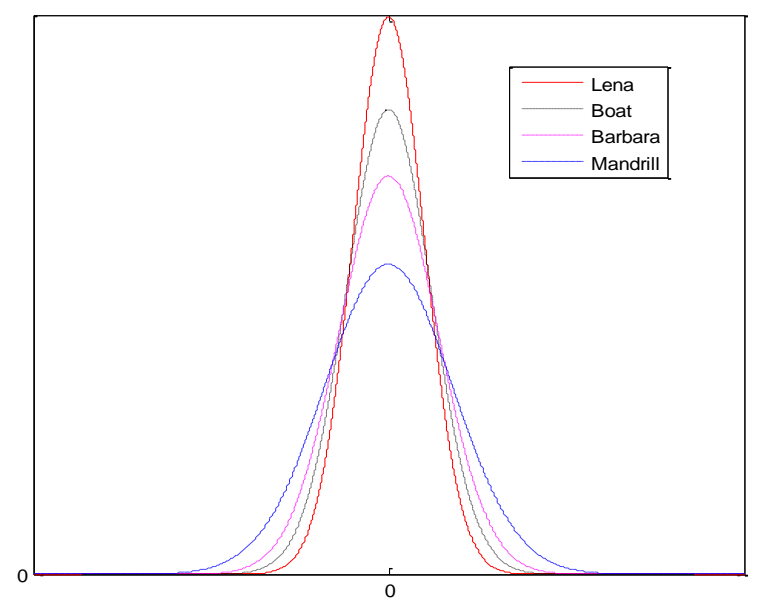

Fig. 3. Generalized Gaussian Distribution for Different Images.

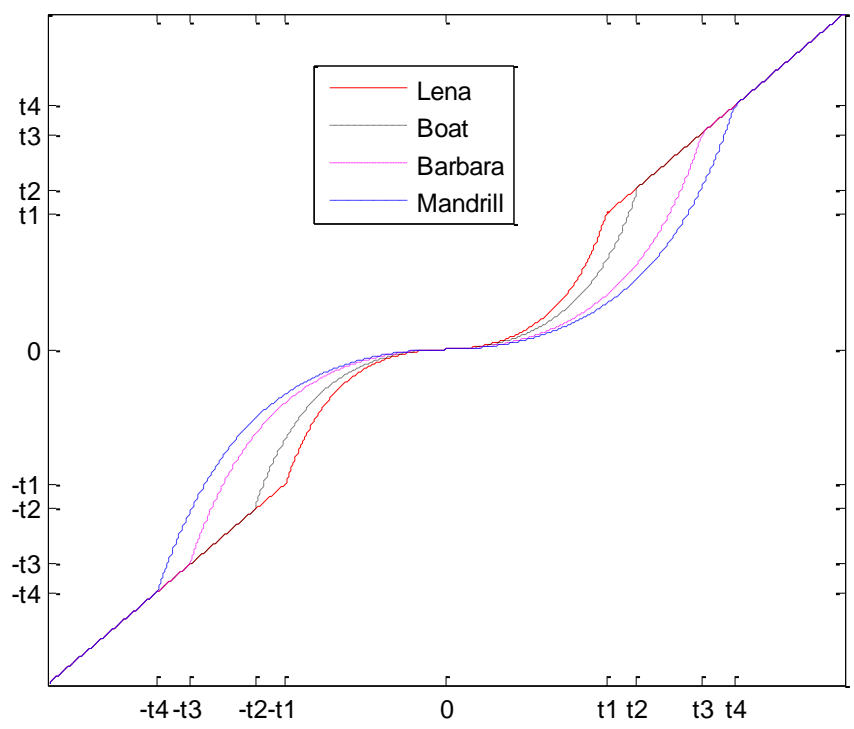

Fig. 4. Proposed Image Dependent Thresholding Functions for four Different Images.

\section{EXPERIMENTAL RESULTS AND DiscuSSIONS}

In this section, in the first experiment, four different images, namely, 'Lena', 'Boat', 'Barbara' and 'Mandrill' $(256 \times 256)$ are used to analyze the performance of the proposed technique along with four state-of-the-art methods available in image de-noising literature. The qualitative results in Fig. 5 shows the superiority of proposed method over Zhang [15], Sahraeian [16] and, Nasri [17]. The visual quality of different de-noising methods for 'Lena', 'Barbara', 'Boat' and 'Mandrill' images are illustrated in this figure. The additive white Gaussian noise with zero mean and standard deviation of 20 is used to generate the corrupted noisy images. PSNR is chosen to be the metric for quantitative analysis. In this context, PSNR results of different de-noising methods for varying standard deviations $\sigma=10,15,20,25$ and 30 are given in Fig. 6. Both qualitative and quantitative results confirm the superiority of proposed method over other state-of-the-art techniques. In this regard another experiment is utilized to show the performance analysis of the proposed method. In this experiment band 20 of Indian Pine hyper-spectral image is utilized. Indian Pine hyper-spectral image is captured by AVIRIS sensor and it consists of $145 \times 145$ pixels in 224 bands. This data set is available in [14]. Fig. 7(a) is the original band 20 of Indian-Pine hyper-spectral image, (b) is the noisy image with PSNR of $21.76 \mathrm{~dB}$, (c) is the de-noised image using smooth sigmoid based shrinkage function (SSBS) proposed in [2] with the PSNR of 30.32 DB and (d) is the denoised image using proposed method with the PSNR of 32.14 $\mathrm{dB}$. 


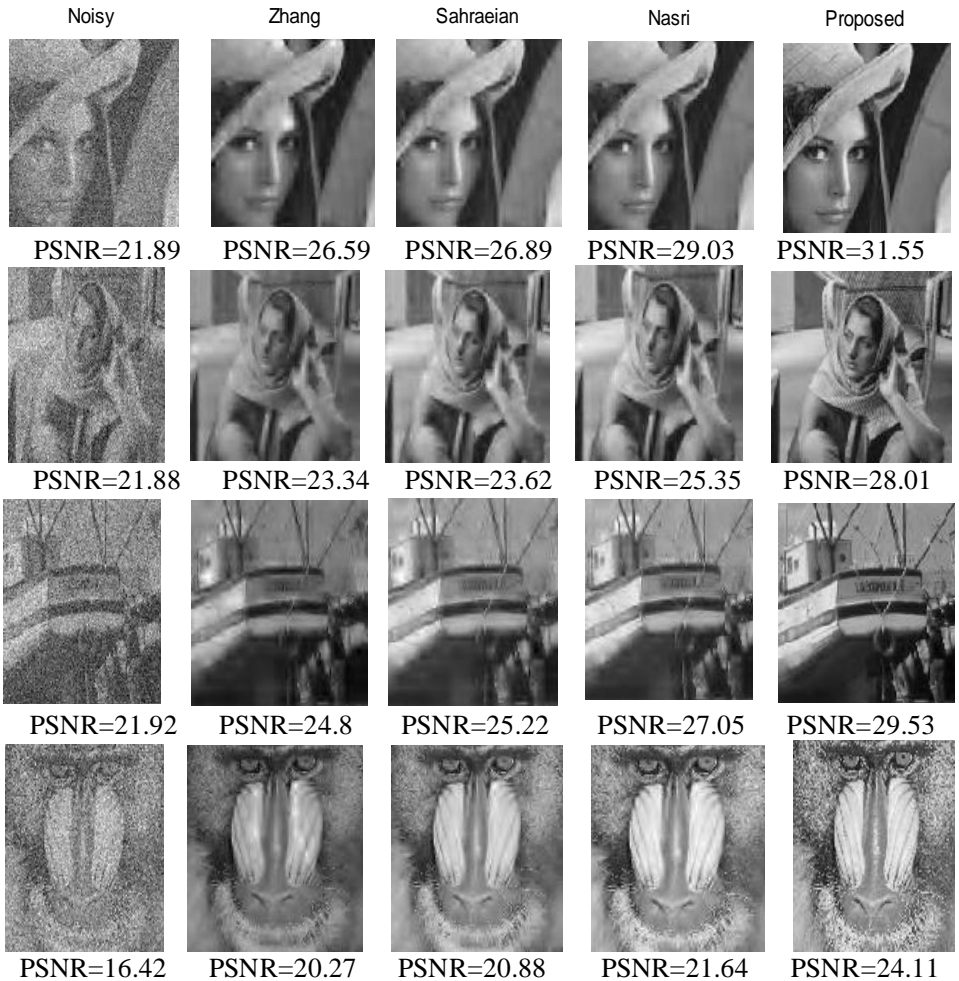

Fig. 5. Comparison of Visual Inspection between Different De-Noising Methods for 'Lena', 'Barbara', 'Boat' and 'Mandrill' Images.

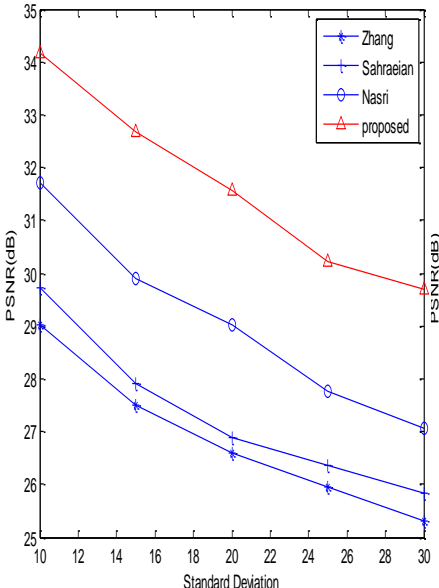

(a)

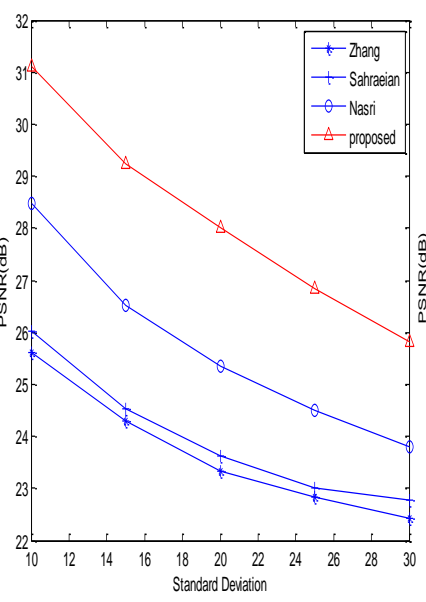

(b)

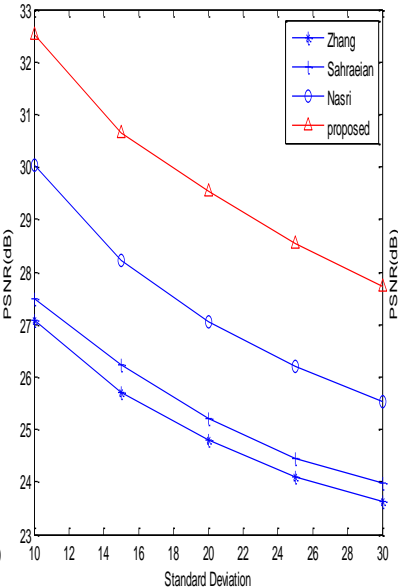

(c)

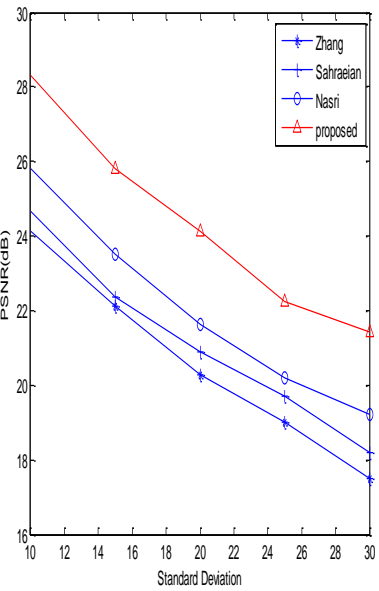

(d)

Fig. 6. Quantitative Results in PSNR, for Varying Noise Variance for Different De-Noising Methods for 'Lena', 'Barbara', 'Boat' and 'Mandrill' Images in (a), (b), (c) and (d), Respectively.

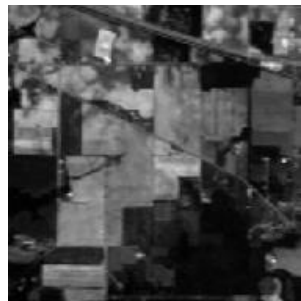

(a)

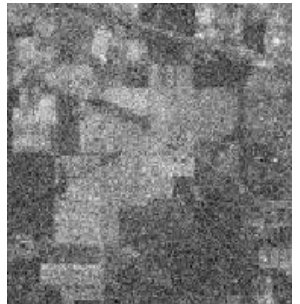

(b)

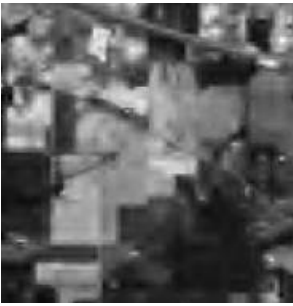

(c)

$P S N R=21.76 \mathrm{~dB}$

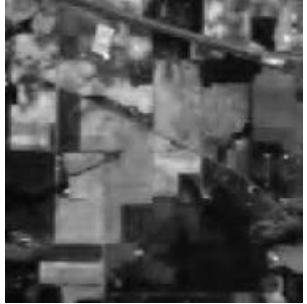

(d)

Fig. 7. De-Noising the Band 20 of Indian Pine Hyper-Spectral Image with Zero Mean and Standard Deviation of 20. 


\section{CONCLUSION}

A new technique for image de-noising utilizing a unique threshold function shaped by a process of using the GGD obtained from the $\mathrm{HH}$ sub-band in the wavelet domain after 4 levels of decomposition is proposed in this paper. Threshold function is formed by using the distribution of the high frequency wavelet coefficients, which makes the function data dependent that is adaptive to the input noisy image. Thresholding is performed in the high frequency sub-bands of the wavelet transform in the interval $[-\mathrm{t}, \mathrm{t}]$, where $t$ is calculated in terms of the standard deviation corresponding to the robust median estimator. After thresholding, inverse wavelet transform is applied to generate the final de-noised image. Visual and quantitative results confirm the superiority of the proposed technique over other alternative state-of-theart methods in the literature. For the future work, it is suggested to work on more nonlinear threshold functions.

\section{REFERENCES}

[1] G.Y. Chen, T.D. Bui, A. Krzyzak, "Image denoising with neighbour dependency and customized wavelet and threshold," Pattern Recogn., vol. 38 pp. 115-124, 2005.

[2] N. A. Golilarz and H. Demirel, "Thresholding neural network (TNN) with smooth sigmoid based shrinkage (SSBS) function for image denoising," in Proceeding of IEEE International Conference on Computational Intelligence and Communication Networks (CICN), (Cyprus), pp. 67-71, (IEEE), Sep 2017.

[3] D.L. Donoho, I.M. Johnstone, "Adapting to unknown smoothness via wavelet shrinkage, ” J. Am. Stat. Assoc., vol. 90, no. 432, pp. 12001224, 1995.

[4] D.L. Donoho and I.M. Johnstone, "Ideal Spatial Adaptation by Wavelet Shrinkage," Biometrika, vol. 81, pp. 425-455, 1993.

[5] J. Portilla, V. Strela, M.Wainwright, E. Simoncelli, "Image de-noising using scale mixture of Gaussians in the wavelet domain, " IEEE Trans. Image Process., vol. 12, no. 11, pp.1338-1350, 2003.

[6] J.R. Sveinsson and J.A. Benediktsson, "Almost translation invariant wavelet transformations for speckle reduction of SAR images," IEEE Trans. Geosci. Remote Sens., vol. 41, no. 10, pp. 2404-2408, 2003.
[7] N. A. Golilarz, H. Gao, W. Ali and M. Shahid, "Hyper-spectral remote sensing image de-noising with three dimensional wavelet transform utilizing smooth nonlinear soft thresholding function," in Proceeding of IEEE International Computer Conference on Wavelet Active Media Technology and Information Processing (ICCWAMTIP), (China), pp. 142-146, (IEEE), Dec 2018.

[8] S. Chang, B. Yu and M. Vetterli, "Adaptive wavelet thresholding for image de-noising and compression," IEEE Trans. Image Processing, pp. 1532-1546, 2000.

[9] D. L. Donoho, "De-noising by soft thresholding," IEEE Trans. Information Theory, vol. 41, no.3, pp. 613-627, 1995.

[10] R. R. Coifman and D. L. Donoho, "Translation-invariant de-noising," in Wavelet and Statistics, Springer Lecture Notes in Statistics 103, New York, Springer-Verlag, pp. 125-150, 1995.

[11] L. Sendur and I. W. Selesnick, "Bivariate shrinkage functions for wavelet based de-noising exploiting inter scale dependency," IEEE Trans. Signal Process., vol. 50, no. 11, pp. 2744-2756, Nov. 2002.

[12] N. A. Golilarz and H. Demirel, "Image de-noising using un-decimated wavelet transform (UWT) with soft thresholding technique," in Proceeding of IEEE International Conference on Computational Intelligence and Communication Networks (CICN), (Cyprus), pp. 1619, (IEEE), Sep 2017.

[13] N. Amiri Golilarz and H. Demirel, "Thresholding neural network (TNN) based noise reduction with a new improved thresholding function," Computational Research Progress in Applied Science and Engineering, vol. 3, no. 2, pp. 81-84, 2017.

[14] B. Rasti, J. R. Sveinsson, M. O. Ulfarsson, J. A. Benediktsson, "Hyperspectral image de-noising using 3D wavelets," in IEEE International Geoscience and Remote Sensing Symposium (IGARSS), (Munich, Germany), pp. 1349-1352, IEEE, Jul 2012.

[15] X. Zhang, "Thresholding neural network for adaptive noise reduction," IEEE Trans. Neural Networks, vol. 12, no. 3, pp. 567-584, May 2001.

[16] S. M. E. Sahraeian, F. Marvasti and N. Sadati, "Wavelet image denoising based on improved thresholding neural network and cycle spinning," in Proceeding of IEEE International Conference on Acoustics, Speech and Signal Processing (ICASSP), (USA), pp.585588, IEEE, April 2007.

[17] M. Nasri and H. Nezamabadi-pour, "Image denoising in the wavelet domain using a new adaptive thresholding function," Neurocomputing, Elsevier, vol. 72, no. 3, pp. 1012-1025, 2009. 